\title{
Propiedades psicométricas del Trait Meta-Mood Scale-24 en estudiantes universitarios argentinos
}

\section{Psychometric properties of the Trait Meta-Mood Scale-24 in Argentinian university students}



Recibido: 20-10-2019 Aceptado: $10-03-2020$

Publicado: 05-06-20

Rocío González iD
Consejo Nacional de Investigaciones Científicas y Técnicas (CONICET). Centro
Interdisciplinario de Investigaciones en Psicología Matemática y Experimental (CIIPME).
Buenos Aires, Argentina
Jesica Brenda Custodio iD - Facundo Juan Pablo Abal iD
Consejo Nacional de Investigaciones Científicas y Técnicas (CONICET). Universidad de
Buenos Aires, Argentina.

Resumen

Introducción: La Inteligencia Emocional Autopercibida (IEA) se conceptualiza como la actitud de las personas hacia su experiencia emocional y la conciencia de sus propias habilidades emocionales. Una de las herramientas más utilizadas para su evaluación es el Trait Meta-Mood Scale (TMMS).

Objetivo: Examinar las propiedades psicométricas del TMMS-24 con el fin de aportar evidencias que garanticen la calidad de la medida en su adaptación al contexto local para su aplicación a estudiantes universitarios de la ciudad de Mar del Plata, Argentina.

Método: La muestra estuvo comprendida por 316 estudiantes (71,5\% mujeres y 28,5\% hombres) de la ciudad de Mar del Plata. Los participantes respondieron un cuestionario sociodemográfico, el Trait Meta-Mood Scale-24 y el Listado de Adjetivos para Evaluar la Personalidad.

Resultados: El análisis factorial exploratorio dio cuenta de un adecuado ajuste del modelo a los datos y una estructura de tres factores (Atención, Claridad y Reparación Emocional), describiendo el 54,5 \% de la varianza. La consistencia interna fue adecuada, observándose coeficientes alfa de Cronbach (entre .82 y .85) y alfa ordinal (entre .84 y .88), muy buenos para las tres sub-escalas. Respecto a la relación con variables sociodemográficas, no se encontraron diferencias según sexo ni asociación con la edad También, se estudió la relación entre IEA y la personalidad.

Conclusiones: El TMMS-24 mostró propiedades psicométricas adecuadas para la medición de IEA en población universitaria marplatense, observándose evidencias de validez (aparente, de contenido, basada en la estructura interna y basada en la relación con otras variables) y de confiabilidad.

Palabras Clave: inteligencia emocional, TMMS-24, propiedades psicométricas, estudiantes universitarios.

\section{Abstract}

Introduction: Perceived Emotional Intelligence (PEI) is conceptualized as people's attitude toward their emotional experience and their awareness of their own emotional abilities. The Trait Meta-Mood Scale (TMMS) is one of the most widely-used tools for its evaluation.

Objective: To test the TMMS-24's psychometric properties to provide evidence that guarantees the measurement's quality when adapting to the local context, so it can be implemented for university students in the city of Mar del Plata, Argentina.

Method: The sample included 316 students (71.5\% women and $28.5 \%$ men) from the city of Mar del Plata. The participants answered a sociodemographic questionnaire, the TMMS-24, and the adjective checklist to assess personality.

Results: The exploratory factor analysis showed that the data adjusted well to the model and showed a three-factor structure (attention, clarity, and emotional healing), describing $54.5 \%$ of the variance. The internal consistency was suitable, showing Cronbach's alpha coefficients (between 0.82 and 0.85 ) and ordinal alpha (between 0.84 and 0.88 ) as very good for the three subscales. Regarding the sociodemographic variables relationship, no differences were found according to sex nor was an association with 
age found. In addition, the relationship between PEI and the personality was studied.

Conclusions: The TMMS-24 showed suitable psychometric properties for PEI measurement among the university students of Mar del Plata. The evidence found was apparent, with content based on the internal structure and relationship with other variables. This evidence was considered valid and reliable.

Keywords: emotional Intelligence, TMMS-24, psychometric properties, university students.

\section{INTRODUCCIÓN}

\subsection{Inteligencia Emocional Autopercibida}

Actualmente, la literatura científica muestra un interés particular en el estudio de las diferencias individuales respecto a las habilidades para procesar y utilizar información emocional (Graesser, 2019; Mayer, Roberts, \& Barsade, 2008; Sosa-Correa, Rodríguez-Ake, Ayuso, Ponce, \& Mestre, 2018). Una de las razones esenciales por las cuales las investigaciones tienden a profundizar en el conocimiento de estos aspectos reside en la importancia que estas habilidades poseen en la experiencia y en el desarrollo humano, considerando, a su vez, su impacto en ciertas áreas como, por ejemplo, la salud y la educación (DeSteno, Gross, \& Kubzansky, 2013).

Es amplio y diverso el avance en teorizaciones acerca de las emociones. Siguiendo los lineamientos de Gardner, basados en la inteligencia intra e interpersonal, surge en la década del noventa el constructo de Inteligencia Emocional (IE). Desde esta perspectiva, se considera a la emoción como un componente necesario para el desarrollo de operaciones mentales, conjuntamente con la motivación y la cognición (Extremera-Pacheco \& FernándezBerrocal, 2016).

Desde la aparición de este constructo, se han elaborado gran número de teorías que se podrían resumir en dos modos de conceptualizar a la IE: aquellas que consideran a la IE como rasgo, entendiéndola como una característica innata que combina habilidades emocionales y rasgos de personalidad, denominadas habitualmente modelos mixtos; y aquellas que entienden a la IE como una habilidad, es decir, como un conjunto de habilidades cognitivas necesarias para el procesamiento de información emocional en contextos intra e interpersonales, similar a la habilidad verbal o matemática (Elfenbein \& MacCann, 2017; Fernández-Berrocal, Ruiz-Aranda, Salguero, Palomera, \& Extremera-Pacheco, 2018; MacCann et al., 2020; Zafra, Martos, \& Martos, 2014).

Entre los modelos mixtos más desarrollados se pueden citar: el modelo de las competencias emocionales de Goleman (1995), el modelo de la IE 
social de Bar-On (1997), y el modelo de IE de Petrides y Furnham (2001). No obstante, estas perspectivas han recibido fuertes críticas basadas en el insuficiente fundamento empírico de sus postulados, en las relaciones con otros constructos pertenecientes al ámbito de la Psicología de la personalidad y, en la forma en que la IE ha sido evaluada a través de cuestionarios de auto-informe (Brackett \& Mayer, 2003; Mikulic, Crespi, \& Caballero, 2018).

Por su parte, el modelo de IE como habilidad que actualmente presenta mayor aceptación en la literatura científica es el de Mayer, Caruso y Salovey (Elfenbein \& MacCann, 2017; Fernández-Berrocal \& Extremera-Pacheco, 2004; MacCann et al., 2020). Estos autores conceptualizan a la IE como la capacidad para identificar, evaluar y diferenciar las emociones propias de las de los demás, saber utilizarlas en la toma de decisiones, comprenderlas y regular tanto emociones positivas como negativas en uno mismo y en otros (Mayer, Caruso, \& Salovey, 1999).

Si bien Mayer y Salovey a partir de 1997 focalizaron sus estudios en la IE Interpersonal (reorganizando el constructo en cuatro dimensiones ordenadas en forma jerárquica: percepción, facilitación, comprensión y regulación emocional), en el inicio de sus trabajos se centraron en la IE Intrapersonal (MacCann et al., 2020; Mayer, Caruso, \& Salovey, 2016). Según estos autores, la Inteligencia Emocional Intrapersonal o Autopercibida (IEA) consiste en las creencias que tienen las personas hacia su experiencia emocional y la conciencia de sus propias habilidades emocionales. De esta manera, esta línea de investigación estudia los procesos cognitivos reflexivos mediante los cuales se perciben, valoran y regulan los estados anímicos de forma constante, denominándose experiencia de meta-humor (Fernández-Berrocal \& Extremera-Pacheco, 2008; Mayer et al., 1999).

Según este modelo, la IEA incluye tres dimensiones que son: la Atención Emocional, la Claridad Emocional y la Reparación Emocional. La Atención Emocional, hace referencia al grado en el que las personas creen prestar atención a sus emociones y sentimientos, es decir, la habilidad para identificar y reconocer los propios sentimientos, así como los estados y sensaciones fisiológicas y cognitivas que estos conllevan. Implica prestar atención y decodificar con precisión las señales emocionales. La Claridad Emocional, se refiere a cómo las personas creen percibir sus emociones, es decir, si experimentan sus sentimientos con claridad y entienden cómo se sienten. Implica desglosar el amplio y complejo repertorio de señales emocionales, discriminar y etiquetar las emociones, y reconocer en qué categorías se agrupan los sentimientos. Por último, la Reparación Emocional, alude a la creencia 
que las personas tienen de su capacidad para interrumpir y regular estados emocionales negativos así como también prolongar estados emocionales positivos. Esta dimensión incluye la capacidad de estar receptivo a los sentimientos y reflexionar sobre los mismos para beneficiarse o descartar la información que los acompaña en función de su utilidad (Extremera-Pacheco \& Fernández-Berrocal, 2005; Salovey, Mayer, Goldman, Turvey, \& Palfai, 1995).

Este modelo resulta de gran interés para la Psicología, ya que se concibe a la $\mathrm{IE}$, no como un rasgo o característica interna que resulta heredable e inmodificable, sino como la puesta en práctica de habilidades y comportamientos susceptibles de aprendizaje y desarrollo, pasibles entonces de entrenamiento (Billings, Downey, Lomas, Lloyd, \& Stough, 2014).

A lo largo de estos años, se han realizado diversas investigaciones empíricas tendientes, no solo a analizar la IEA, sino también las particularidades de este concepto en distintos ámbitos, tales como el educativo, laboral, familiar y de salud mental (Cejudo, López-Delgado, \& Rubio, 2016; Petrides et al., 2016; Rincón \& Rodríguez, 2018). En líneas generales, la mayoría de estos estudios evidencian que las personas emocionalmente inteligentes presentan un adecuado ajuste psicológico y social que se manifiesta a partir de la presencia de una serie de indicadores. Entre ellos, se pueden mencionar, por ejemplo, respuestas adaptativas ante situaciones vitales negativas; bajos niveles de estrés, emocionalidad negativa y alexitimia; puntuaciones bajas en sintomatología psicológica (especialmente en ansiedad y depresión); adecuados niveles de satisfacción vital, empatía y optimismo; relaciones interpersonales satisfactorias; rendimiento y satisfacción académica apropiados (Cejudo et al., 2016; Fernández-Berrocal \& Extremera-Pacheco, 2008; Kotsou, Mikolajczak, Heeren, Grégoire, \& Leys, 2019; MacCann et al., 2020; Sosa-Correa et al., 2018).

Específicamente en el ámbito educativo, se ha observado que la IE es una habilidad importante en los estudiantes. Siendo un constructo no-cognitivo, tiene poder predictivo en cuanto al rendimiento académico y el desarrollo personal (Barna \& Brott, 2011; MacCann et al., 2020). De hecho, carecer de ciertas habilidades propias de la IE afecta a los estudiantes dentro y fuera del contexto educativo. Contar con adecuadas habilidades emocionales favorece el pensamiento, incrementa la concentración y la motivación intrínseca, permite el control de la conducta impulsiva, posibilita la adaptación educativa y el desarrollo de estrategias de afrontamiento ante el estudio. A su vez, facilita el establecimiento de relaciones interpersonales, un rendimiento adecuado en condiciones de estrés y se asocia a indicadores de bienestar 
psicológico (Belmonte-Lillo, 2013; Cejudo et al., 2016; Delgado, MartínezMonteagudo, Rodríguez, \& Escortell-Sánchez, 2019; Molero, et al., 2020).

En las últimas décadas, debido a los elevados niveles de estrés, ansiedad y fracaso escolar presentes en el ámbito educativo, la IE ha adquirido una gran relevancia. Su entrenamiento y desarrollo propicia el bienestar psicológico de los estudiantes, facilitándoles la comprensión del entorno que los rodea, así como también dotándolos de competencias necesarias para hacer frente a las diversas situaciones que se presentan a lo largo de su recorrido académico (Molero, et al., 2020).

\subsection{Medición de la Inteligencia Emocional Autopercibida}

Entre los diversos instrumentos que existen para medir la IEA, el Trait Meta-Mood Scale -TMMS- se destaca por ser una de las herramientas más utilizadas a nivel mundial (Fernández-Berrocal, Berrios-Martos, ExtremeraPacheco, \& Augusto, 2012; Mikulic et al., 2018; Rincón \& Rodríguez, 2018). Permite obtener un índice que valora el conocimiento que cada persona posee acerca de sus propios estados emocionales, brindando una estimación personal sobre los aspectos reflexivos de la experiencia emocional (ExtremeraPacheco \& Fernández-Berrocal, 2005).

El TMMS, en su versión original que consta de 48 ítems, permite diferenciar tres dimensiones para la IEA: a) Atención Emocional, compuesta por 21 ítems (p.e. "Pienso en mi estado de ánimo constantemente"); b) Claridad Emocional con 15 ítems (p.e. "Frecuentemente me equivoco con mis sentimientos"); y c) Reparación Emocional, constituida por 12 ítems (p.e. "Aunque a veces me siento triste, suelo tener una visión optimista") (Salovey et al., 1995).

Fernández-Berrocal et al. (1998) realizaron una adaptación al español del TMMS-48, encontrando propiedades psicométricas muy similares a las de la escala original. Años más tarde, Fernández-Berrocal, Extremera-Pacheco y Ramos (2004) desarrollaron una versión reducida y adaptada al español de esta escala americana, denominándola Trait Meta-Mood Scale-24 -TMMS24-. Esta versión mantiene las tres dimensiones originales de la escala, pero reduce los ítems a la mitad, conservando aquellos que maximizan la consistencia interna. De esta manera, la escala final de esta versión española está compuesta por 24 ítems, con ocho ítems por dimensión. Al presentar propiedades psicométricas adecuadas, se recomienda la utilización en castellano de esta versión reducida (Extremera-Pacheco \& Fernández-Berrocal, 2005). 
Esta versión breve del TMMS fue traducida y adaptada a otros idiomas y culturas (Fernández-Berrocal \& Extremera-Pacheco, 2008) como por ejemplo: alemán (Otto, Döring-Seipel, Grebe, \& Lantermann, 2001), portugués (BritoCosta et al., 2016), francés (Bourdier \& Ringuenet, 2016), vasco (Gorostiaga, Balluerka, Aritzeta, Haranburu, \& Alonso-Arbiol, 2011), turco (Aksöz, Bugay, \& Erdur-Baker, 2010), chino (Li, Yan, Yin, \& Wu, 2002), italiano (Giromini, Colombarolli, Brusadelli, \& Zennaro, 2017), mexicano (Valdivia-Vázquez, Rubio-Sosa, \& French, 2015).

En Argentina, hasta la fecha, se ha desarrollado un estudio psicométrico preliminar del TMMS-48 (Mikulic, Crespi, Caballero, Aruanno, \& Paolo, 2017). Además, se realizó la construcción y validación de un instrumento para evaluar Inteligencia Emocional Percibida en adultos partiendo del modelo de Salovey y Mayer, pero considerando no solo las habilidades intrapersonales sino también las interpersonales (Mikulic et al., 2018). También, se ha publicado una versión del TMMS de 21 ítems para adolescentes (Calero, 2013).

Tomando en consideración lo antes mencionado, el objetivo del presente estudio consiste en examinar las propiedades psicométricas del Trait Meta-Mood Scale-24 con el fin de aportar evidencias que garanticen la calidad de la medida en su adaptación al contexto local para su aplicación a estudiantes universitarios de la ciudad de Mar del Plata, Argentina.

En síntesis, una serie de razones dan cuenta de la relevancia de este trabajo:

1) La IE en el ámbito universitario facilita tanto el proceso de formación como el logro de las expectativas de éxito profesional de los futuros egresados. El contexto laboral demanda de ellos, una vez graduados, no solo saberes, sino también ser portadores de habilidades socioemocionales que les permitan afrontar exitosamente problemáticas laborales futuras (Rodríguez, Sánchez, Ochoa, Cruz, \& Fonseca, 2019). Por este motivo, es esencial contar con instrumentos que permitan valorar este constructo.

2) A su vez, es sumamente relevante realizar este tipo de estudios para examinar el modo de presentación de esta variable en nuestro contexto sociocultural, ya que podría ser un factor mediador de la experiencia subjetiva asociada a las emociones. El contexto puede modificar tanto el significado de la experiencia emocional como la expresión emocional, es decir, el modo en que se comunican y manifiestan las emociones (Fernández, Carrera, \& Sánchez, 2001). Cada cultura tiene sus propias 
reglas de expresión emocional que se adquieren por medio del aprendizaje y modulan el significado de las emociones. De este modo, la cultura atraviesa e influye la manera en que las emociones son interpretadas, intensificando, disminuyendo, sustituyendo o neutralizando su aparición y/o expresión (Ekman, Sorenson, \& Friesen, 1969).

3) Contar con instrumentos debidamente adaptados a nuestro medio permite realizar comparaciones válidas con los resultados obtenidos en otros países.

4) A esto se suma, el hecho de que existen escasos estudios que den cuenta de cómo funciona este instrumento en estudiantes universitarios argentinos. Disponer de una herramienta adecuada para valorar la IEA en esta población permitiría evaluar, entre otros aspectos, el impacto de intervenciones educativas dirigidas a promover estados emocionales más funcionales para afrontar con éxito las exigencias que los estudiantes atraviesan para alcanzar los objetivos académicos (Domínguez-Lara \& Medrano, 2016).

\section{MÉTODO}

\subsection{Diseño}

Se corresponde a un estudio de tipo instrumental según los criterios de clasificación propuestos por Ato, López y Benavente (2013). Se trata de un estudio de corte psicométrico tendiente a la obtención de evidencias de validez y confiabilidad de la adaptación local del TMMS-24 en población universitaria.

\subsection{Participantes}

Colaboraron un total de 323 estudiantes de ambos sexos de distintas carreras de la Universidad Pública de la ciudad de Mar del Plata (Buenos Aires, Argentina), que fueron seleccionados mediante un muestreo no probabilístico e intencional. Siete individuos fueron eliminados en los análisis preliminares, por lo que la muestra definitiva contó con 316 participantes.

En relación con la edad, se evaluó a sujetos entre 18 y 54 años que accedieron voluntariamente a participar de la investigación con previa autorización (Edad: $M=21.02$; $D E=3.71$ ). Respecto al sexo, el $71,5 \%$ de la muestra estuvo conformada por mujeres y el $28,5 \%$ restante por varones.

Con el propósito de lograr una muestra heterogénea, fueron incluidos estudiantes de diferentes carreras. El 23,4\% de los estudiantes asistía a la Facultad de Psicología, el 19,9 \% a la Facultad de Ciencias Económicas y 
Sociales, el 20,6 \% a la Facultad de Ciencias Exactas y Naturales, el 17,7 \% a la Facultad de Arquitectura, Urbanismo y Diseño, y por último el $18,4 \%$ a la Facultad de Derecho. Respecto al nivel socio-económico autopercibido, el $0,9 \%$ de los estudiantes refirió tener un nivel bajo, el $61,4 \%$ un nivel medio y el $31,7 \%$ un nivel alto. Un $6 \%$ de la muestra omitió responder este aspecto.

\subsection{Instrumentos}

\subsubsection{Cuestionario sociodemográfico}

Se trata de un cuestionario auto-administrado construido ad hoc para obtener datos sociodemográficos de la muestra, tales como sexo, edad, lugar de residencia y nivel socio-económico autopercibido.

\subsubsection{Trait Meta-Mood Scale-24 (TMMS-24; Fernández-Berrocal et al.,} 2004)

Se trata de una medida de auto-informe que evalúa la IEA basada en el modelo de Salovey y Mayer (1990). Este cuestionario está conformado por 24 ítems con ocho reactivos por cada sub-escala respectivamente: a) Atención Emocional, b) Claridad Emocional y c) Reparación Emocional. El formato de respuesta consiste en una escala tipo Likert que presenta cinco opciones (1. totalmente en desacuerdo; 2 . en desacuerdo; 3 . ni de acuerdo ni en desacuerdo; 4. de acuerdo; 5 . totalmente de acuerdo). En la consiga, se pide a la persona que indique el grado de acuerdo con cada afirmación sobre sus emociones y sentimientos. Para obtener la puntuación en cada uno de los factores, se suman los ítems del 1 al 8 para el factor Atención Emocional, los ítems del 9 al 16 para el factor Claridad Emocional y del 17 al 24 para el factor Reparación Emocional (todos en orden directo). Las propiedades psicométricas de la versión española muestran que para cada factor, la consistencia interna es aceptable ( $\alpha=.90$ para Atención Emocional; $\alpha=.90$ para Claridad Emocional y $\alpha=.86$ para Reparación Emocional) y la fiabilidad test-retest es adecuada (Atención Emocional $=.60$; Claridad Emocional $=.70$ y Reparación Emocional $=.83$ ). Los tres factores correlacionan de forma apropiada con variables de criterio clásicas tales como depresión, ansiedad, rumiación y satisfacción vital (Fernández-Berrocal et al., 2004).

\subsubsection{Listado de Adjetivos para Evaluar la Personalidad (AEP; Ledesma,} Sánchez, \& Díaz-Lázaro, 2011)

Consiste en una lista de 67 adjetivos con cinco opciones de respuesta (desde 1. no me describe en absoluto hasta 5. me describe tal como soy) para evaluar las cinco dimensiones del modelo de los Cinco Grandes de la personalidad 
(Extraversión, Amabilidad, Responsabilidad, Neuroticismo y Apertura). Este instrumento fue desarrollado y validado en la ciudad de Mar del Plata y posee propiedades psicométricas adecuadas. Los valores $\alpha$ de Cronbach oscilan entre .75 y .84 para las diferentes escalas. A su vez, la estructura factorial resulta acorde a las dimensiones propuestas por el modelo.

\subsection{Procedimiento}

El procedimiento seguido ha contemplado las normativas profesionales internacionales para la adaptación y la validación de tests usados en la práctica clínica e institucional (American Educational Research Association, American Psychological Association y National Council on Measurement in Education, 2014; American Psychological Association, 2010) y, más específicamente, en investigación psicológica (International Test Commission, 2014). Asimismo, el protocolo fue aprobado por el Comité de Conductas Responsables de la Facultad de Psicología de la Universidad de Buenos Aires, sede en la que se desarrolla uno de los dos proyectos de investigación en el que se enmarca el presente estudio (UBACyT2018 20020170200001BA). El otro proyecto de investigación corresponde al Instituto de Psicología Básica, Aplicada y Tecnología de la Facultad de Psicología de la Universidad Nacional de Mar del Plata.

Inicialmente, se contactó a las autoridades de las facultades intervinientes, se les explicó el objetivo del estudio y se obtuvo la autorización para llevar adelante la investigación. El contacto con los estudiantes se realizó por medio del Grupo de Investigación en Psicología Cognitiva y Educacional que tiene su sede de trabajo en la Facultad de Psicología de la Universidad Nacional de Mar del Plata (Buenos Aires, Argentina). Siguiendo los principios éticos vigentes, se solicitó a los estudiantes la firma del consentimiento informado como condición excluyente para participar del estudio. Además, se les informó acerca de los objetivos de este trabajo, la confidencialidad de los datos recolectados y se les aclaró que su participación era voluntaria, pudiendo abandonar la investigación en el momento que lo desearan.

A continuación, los estudiantes respondieron los protocolos de modo autoadministrado, en formato grupal, y en el aula, en forma previa o posterior a su horario regular de clases. La administración fue supervisada por profesionales idóneos y habilitados para tal fin.

\subsection{Análisis de Datos}

Con el fin de obtener evidencias de validez de contenido se recurrió a un estudio de juicio experto para que dictamine sobre la adecuación lingüística y 
conceptual de la versión española del instrumento (Fernández-Berrocal et al., 2004) al ser aplicada en el contexto local. La información obtenida se complementó con un estudio piloto, que aportó evidencias de validez aparente a la adaptación.

Luego de recolectar los casos, se procedió a un análisis descriptivo de los 24 ítems. Cabe destacar que debido a que los protocolos fueron revisados antes de ser entregados, no se identificaron datos perdidos, por lo cual no fue necesario llevar a cabo imputaciones.

Seguidamente, se realizó un análisis preliminar de los datos a modo exploratorio (Uriel \& Aldas, 2005) para verificar los supuestos estadísticos y también, detectar casos atípicos que pudieran distorsionar los resultados.

A continuación, se realizó un Análisis Factorial Exploratorio (AFE) con el programa FACTOR 9.3 (Lorenzo-Seva \& Ferrando, 2015) para aportar evidencias de validez basada en la estructura interna. Este análisis permitió examinar si se mantenía la estructura interna de la escala original y de la versión española. Para la realización del AFE, se han seguido las pautas sugeridas por la literatura actual (Lloret-Segura, Ferreres-Traver, HernándezBaeza, \& Tomás-Marco, 2014), que recomiendan el uso de correlaciones policóricas en lugar de correlaciones de Pearson en aquellas variables que no presenten distribución normal (Abad, Olea, Ponsoda, \& García, 2011). Además, se sugiere su uso cuando el formato de respuesta es de tipo Likert (ordinales). Respecto al tamaño de la muestra, se ha considerado contar con no menos de 10 participantes por ítem para disminuir el error de muestreo y evitar la inestabilidad de los factores (Tabachnick \& Fidell, 2001).

Posteriormente, se analizó la confiabilidad a través de un análisis de consistencia interna de los ítems de cada una de las sub-escalas por medio del cálculo de los coeficientes $\alpha$ de Cronbach y $\alpha$ ordinal con sus respectivos intervalos de confianza del 95 \%. Este análisis se realizó con el programa R utilizando la función scaleReliability del paquete userfriendlyscience (Peters, 2014).

Por último, se analizó la posible influencia de variables sociodemográficas. Para ello, se evaluó la existencia de diferencias según sexo por medio de una 
prueba $U$ de Mann Whitney, y se estudió la asociación con la edad por medio de una correlación bivariada de Spearman.

Además, con el objetivo de aportar evidencias de validez basada en la relación con otras variables, se estudiaron las correlaciones entre las puntuaciones de las sub-escalas del TMMS-24 (Atención Emocional, Claridad Emocional y Reparación Emocional) y del AEP (Extraversión, Amabilidad, Responsabilidad, Neuroticismo y Apertura), por medio de una correlación bivariada de Spearman.

\section{RESULTADOS}

\subsection{Adaptación lingüística y estudio piloto: validez de contenido y} validez aparente

El proceso de adaptación se realizó teniendo en cuenta las directrices de la Internacional Tests Commissión (2014). Si bien se ha realizado la adaptación y validación del TMMS-48 para su uso en adultos de Buenos Aires (Mikulic et al., 2017), no se conocen propiedades psicométricas del TMMS-24 en nuestro medio, puesto que no se han desarrollado estudios a nivel local. Sin embargo, como ya existe una versión del TMMS-24 en castellano, además de la versión original (Salovey et al., 1995), se decidió realizar los ajustes lingüísticos sobre la versión española del instrumento (Fernández-Berrocal et al., 2004).

Con el fin de lograr frases adecuadas localmente, en el ítem 4 ("Pienso que merece la pena prestar atención a mis emociones y estado de ánimo"), se cambió la palabra merece por vale. Y también, en el ítem 24 (“Cuando estoy enfadado intento cambiar mi estado de ánimo"), se resolvió modificar la palabra enfadado por enojado. En el resto de los ítems se conservó la redacción de la versión española porque no se empleaban regionalismos que llevaran a dificultades de comprensión.

El instrumento fue enviado a cinco jueces expertos para que evaluaran a ciegas la adecuación a nivel formal (sintáctico y semántico) de cada uno de los ítems, así como también la pertenencia de cada ítem a las sub-escalas referidas por los autores. Para este último aspecto, se evaluó el grado de acuerdo entre jueces mediante el cálculo del índice $V$ de Aiken y su intervalo de confianza del $90 \%$. Se adoptó como criterio de aceptación un límite inferior 
del intervalo de confianza del $V$ de Aiken $\geq .60$, valor que no fue superado por ninguno de los ítems (Tabla 1).

Por último, la versión final del instrumento fue sometida a una prueba piloto con 10 estudiantes universitarios, varones y mujeres de diferentes edades, para evaluar la compresión y adecuación de los ítems, de la consigna y del formato de respuesta. Se arribó así, a una adaptación lingüística y conceptual del instrumento que permitió aportar evidencias de validez aparente y de contenido.

\subsection{Análisis preliminar de los datos}

Se calculó la distancia de Mahalanobis considerando como punto de corte $\mathrm{p} \leq .001$ (Tabachnick \& Fidell, 2001), con el fin de explorar la presencia de casos anómalos multivariantes. Se detectaron 7 outliers que debieron ser eliminados para los posteriores análisis. Por ende, el tamaño muestral definitivo fue de 316 participantes. También se analizaron los casos atípicos univariados a partir del cálculo de un puntaje $Z$ para cada uno de los ítems. Se observaron 33 datos con valores fuera del rango $Z \pm 3$ (Tabachnick \& Fidell, 2001). Estos casos se corresponden con los ítems 1 ("Presto mucha atención a los sentimientos"), 4 ("Pienso que vale la pena prestar atención a mis emociones y estado de ánimo"), 13 ("A menudo me doy cuenta de mis sentimientos en diferentes situaciones") y 23 ("Tengo mucha energía cuando me siento feliz"). Dado que se optó por no eliminarlos, se tuvo cautela al momento de su interpretación (Hair, Black, Babin, \& Anderson, 2009).

Luego, se realizó un análisis descriptivo de los 24 ítems calculando sus medias, desviaciones estándar e índices de asimetría y curtosis. En la Tabla 1 se puede observar que siete de los ítems exceden los valores \pm 1 de asimetría y/o curtosis recomendados por George y Mallery (2016) como criterio para aceptar la normalidad de las variables. Se destaca particularmente la distribución del ítem 23, cuyo contenido refleja un indicador básico y esencial de la dimensión Reparación Emocional. Como consecuencia, la mayoría de los sujetos evaluados tendieron a concentrarse en la categoría 5.

Por último, se examinaron los diagramas de dispersión mediante un análisis visual para evaluar la existencia de relaciones lineales entre las variables analizadas, y se realizó un estudio de la multicolinealidad entre los ítems para 
identificar correlaciones demasiado altas o redundantes (Tabachnick \& Fidell, 2001). No se detectaron correlaciones inter-ítem iguales o superiores a \pm .90 .

Tabla 1.

Acuerdo entre jueces y estadísticos descriptivos de los ítems del TMMS-24

\begin{tabular}{|c|c|c|c|c|c|c|}
\hline SUB-ESCALAS IEA & ÍTEMS & $\begin{array}{l}\text { V DE AIKEN } \\
\text { [90IC] }\end{array}$ & M & DE & ASIMETRÍA & CURTOSIS \\
\hline \multirow{8}{*}{ Atención Emocional } & IE1 & $1[.79-1]$ & 4.15 & 0.94 & -1.20 & 1.26 \\
\hline & IE2 & $.90[.65-.97]$ & 3.96 & 1.06 & -0.97 & 0.43 \\
\hline & IE3 & $.90[.65-.97]$ & 3.62 & 1.18 & -0.67 & -0.36 \\
\hline & IE4 & $1[.79-1]$ & 4.19 & 0.96 & -1.30 & 1.61 \\
\hline & IE5 & $.90[.65-.97]$ & 3.28 & 1.33 & -0.33 & -1.03 \\
\hline & IE6 & $.90[.65-.97]$ & 2.92 & 1.34 & 0.02 & -1.17 \\
\hline & IE7 & $1[.79-1]$ & 3.53 & 1.13 & -0.60 & -0.35 \\
\hline & IE8 & $1[.79-1]$ & 3.60 & 1.11 & -0.58 & -0.36 \\
\hline \multirow{8}{*}{ Claridad Emocional } & IE9 & $1[.79-1]$ & 3.38 & 1.20 & -0.35 & -0.75 \\
\hline & IE10 & $1[.79-1]$ & 3.39 & 1.17 & -0.52 & -0.60 \\
\hline & IE11 & $1[.79-1]$ & 3.56 & 1.08 & -0.55 & -0.34 \\
\hline & IE12 & $.90[.65-.97]$ & 3.96 & 1.00 & -0.93 & 0.55 \\
\hline & IE13 & $1[.79-1]$ & 3.98 & 0.94 & -1.03 & 1.09 \\
\hline & IE14 & $1[.79-1]$ & 3.20 & 1.26 & -0.17 & -0.97 \\
\hline & IE15 & $.90[.65-.97]$ & 3.66 & 1.08 & -0.69 & -0.02 \\
\hline & IE16 & $1[.79-1]$ & 3.62 & 1.02 & -0.62 & -0.01 \\
\hline \multirow{8}{*}{ Reparación Emocional } & IE17 & $.90[.65-.97]$ & 3.70 & 1.34 & -0.81 & -0.54 \\
\hline & IE18 & $.90[.65-.97]$ & 3.65 & 1.27 & -0.70 & -0.52 \\
\hline & IE19 & $1[.79-1]$ & 3.03 & 1.40 & -0.12 & -1.23 \\
\hline & IE20 & $1[.79-1]$ & 3.68 & 1.28 & -0.72 & -0.54 \\
\hline & IE21 & $.90[.65-.97]$ & 3.76 & 1.25 & -0.77 & -0.46 \\
\hline & IE22 & $1[.79-1]$ & 3.92 & 1.03 & -0.84 & 0.22 \\
\hline & IE23 & $.90[.65-.97]$ & 4.66 & 0.77 & -2.86 & 8.73 \\
\hline & IE24 & $1[.79-1]$ & 3.41 & 1.25 & -0.48 & -0.71 \\
\hline
\end{tabular}

Nota. M: Media; DE: Desvío Estándar.

3.3. Evidencias de validez basada en la estructura interna: análisis factorial exploratorio

Se realizó un AFE con el fin de aportar evidencias de validez basada en la estructura interna del instrumento. Previamente, se calcularon indicadores que permitan sostener la factibilidad de este estudio multivariante: el test de esfericidad de Bartlett y la medida de adecuación muestral de Kaiser-Mayer- 
Olikin (KMO). Tanto un KMO satisfactorio de .85 como la prueba Bartlett ( $\mathrm{X}^{2}$ $=3081.3 ; \mathrm{gl}=276 ; \mathrm{p}<0.001$ ) sugieren la posibilidad de aplicar un análisis factorial a la matriz de datos.

La implementación óptima del análisis paralelo de Horn (basado en el método de extracción de mínimos cuadrados no ponderados), realizado sobre la matriz de correlaciones policóricas de los 24 ítems, sugirió la extracción de tres factores. Otros criterios objetivos, como GFI $=.98$, RMSEA $=.04$ y RMSR $=0.055$, indicaron un buen ajuste del modelo a los datos, complementando la decisión de retener tres factores (Abad et al., 2011; Lloret-Segura et al., 2014).

Dado que los factores aislados mostraron una correlación considerable (superior a .32), se decidió la aplicación de un método de rotación oblicua (Promin). Los 24 ítems se distribuyeron, conforme se esperaba a nivel teórico, de manera equilibrada en tres factores que describieron el 54,5\% de la varianza. El índice de simplicidad factorial de Bentler alcanzó un valor adecuado $(S=0.99$ ). Tal lo expuesto en la Tabla 2 , cada factor presenta al menos cuatro ítems y, a su vez, todos los ítems presentaron cargas factoriales superiores a .30 .

Los ítems que saturaron en el primer factor son indicativos de la sub-dimensión Claridad Emocional (ser capaz de comprender adecuadamente los estados emocionales). El segundo factor estuvo compuesto por ítems propuestos para la sub-dimensión Atención Emocional (ser capaz de atender a los sentimientos de forma adecuada). Por último, los elementos que componen el tercer factor operacionalizan la sub-dimensión Reparación Emocional (ser capaz de regular los estados emocionales adecuadamente). Solo la correlación entre los factores Claridad Emocional y Reparación Emocional fue moderada $(r=.45)$. Atención Emocional, en cambio, mostró una baja asociación con Claridad Emocional ( $r=.17$ ) así como con Reparación Emocional $(r=.07)$.

Tabla 2.

Cargas factoriales de los reactivos del TMMS-24 (matriz rotada) y consistencia interna

\begin{tabular}{cccc}
\hline \multirow{2}{*}{ ÍTEMS } & \multicolumn{3}{c}{ FACTOR } \\
\cline { 2 - 4 } & I & II & III \\
\hline IE1 & .72 & \\
IE2 & .79 & \\
IE3 & .68 &
\end{tabular}




\begin{tabular}{|c|c|c|c|}
\hline \multirow{2}{*}{ ÍTEMS } & \multicolumn{3}{|c|}{ FACTOR } \\
\hline & I & II & III \\
\hline IE4 & & .62 & \\
\hline IE5 & & .51 & \\
\hline IE6 & & .67 & \\
\hline IE7 & & .68 & \\
\hline IE8 & & .76 & \\
\hline IE9 & .59 & & \\
\hline IE10 & .73 & & \\
\hline IE11 & .79 & & \\
\hline IE12 & .60 & & \\
\hline IE13 & .59 & & \\
\hline IE14 & .75 & & \\
\hline IE15 & .50 & & \\
\hline IE16 & .57 & & \\
\hline IE17 & & & .83 \\
\hline IE18 & & & .91 \\
\hline IE19 & & & .75 \\
\hline IE20 & & & .90 \\
\hline IE21 & & & .60 \\
\hline IE22 & & & .62 \\
\hline IE23 & & & .33 \\
\hline IE24 & & & .60 \\
\hline$\alpha$ Cronbach [95 IC] & $.82[.79, .85]$ & $.84[.81, .86]$ & $.85[.83, .88]$ \\
\hline$\alpha$ ordinal [95 IC] & $.84[.81, .87]$ & $.87[.85, .89]$ & $.88[.86, .90]$ \\
\hline
\end{tabular}

Nota. Se omitieron cargas inferiores a .30

\subsection{Análisis de la confiabilidad}

Al estudiar la confiabilidad a través de un análisis de la consistencia interna de los ítems, se verificaron coeficientes alfa de Cronbach muy buenos para las tres sub-escalas del instrumento (Tabla 2): atención emocional $=.82$; claridad emocional $=.84$; reparación emocional $=.85$. Los índices resultan ligeramente más elevados si se respeta el carácter politómico de las categorías de respuesta a los ítems calculando el alfa ordinal.

\subsection{Relación con características sociodemográficas}

A continuación, se realizó una prueba U de Mann Whitney, comparando las puntuaciones obtenidas por varones y mujeres en cada una de la sub-es- 
calas. Se optó por una prueba no paramétrica dado que no se cumplían los supuestos de normalidad y homocedasticidad. No se observaron diferencias estadísticamente significativas en ninguna de ellas en función del sexo (Tabla 3).

Tabla 3.

Diferencias en función del sexo

\begin{tabular}{ccccc}
\hline & \multicolumn{2}{c}{ SEXO RANGO PROMEDIO } & & \\
\cline { 2 - 5 } SUB-ESCALAS & $\begin{array}{c}\text { VARONES } \\
\text { (N }=\mathbf{9 0})\end{array}$ & $\begin{array}{c}\text { MUJERES } \\
\text { (N= 226) }\end{array}$ & $\begin{array}{c}\text { U DE } \\
\text { MANN WHITNEY }\end{array}$ & P \\
\hline Claridad Emocional (F1) & 145.84 & 163.54 & 11309.50 & .12 \\
Atención Emocional (F2) & 173.97 & 152.34 & 8777.50 & .06 \\
Reparación Emocional (F3) & 164.54 & 156.09 & 9626 & .46 \\
\hline Nota. ${ }^{*}$ p<.05; ${ }^{* *} p<.01 ; * * * 0.001$ (bilateral) & & &
\end{tabular}

Además, para identificar si existía asociación entre la edad de los participantes y las sub-escalas del TMMS-24 se correlacionaron las variables mediante el coeficiente Rho de Spearman. Se utilizó una prueba no paramétrica dado la asimetría de las variables. No se hallaron asociaciones estadísticamente significativas con ninguna de los tres sub-escalas (Atención Emocional: $r=$ $.08, p=.15$; Claridad Emocional: $r=.05, p=.39$; Reparación Emocional: $r$ $=.04, p=.51)$.

\subsection{Evidencias de validez basada en la relación con otras variables}

Se realizaron correlaciones entre las sub-escalas del TMMS-24 y del AEP. En la Tabla 4, se puede observar que todas las correlaciones resultaron ser significativas, excepto Responsabilidad/Reparación Emocional, Responsabilidad/ Claridad Emocional, y Extraversión/Claridad Emocional. Las correlaciones significativas fueron positivas en todos los casos, excepto Neuroticismo/ Atención Emocional y Neuroticismo/Reparación Emocional.

Tabla 4.

Correlaciones entre sub-escalas del TMMS-24 y sub-escalas del AEP

\begin{tabular}{ccccccc}
\hline & \multicolumn{5}{c}{ PERSONALIDAD } \\
\hline & EXTRAVERSIÓN & AMABILIDAD & RESPONSABILIDAD & NEUROTICISMO & APERTURA \\
\hline \multirow{3}{*}{ IEA $\quad$ Claridad Emocional } & $.30^{* *}$ & $.26^{* *}$ & $.17^{* *}$ & $-.33^{* *}$ & $.19^{* *}$ \\
& .08 & $.23^{* *}$ & -.07 & $.24^{* *}$ & $.13^{*}$ \\
& Reparación Emocional & $.37^{* *}$ & $.34^{* *}$ & .09 & $-.43^{* *}$ & $.21^{* *}$ \\
\hline
\end{tabular}

Nota. ${ }^{*} p<.05 ;{ }^{* *} p<.01 ; * * p<.001$ (bilateral) 


\section{DISCUSIÓN}

El objetivo del presente estudio consistió en examinar las propiedades psicométricas del Trait Meta-Mood Scale-24 con el fin de aportar evidencias que garanticen la calidad de la medida en su adaptación al contexto local para su aplicación a estudiantes universitarios de la ciudad de Mar del Plata, Argentina.

En primer lugar, se han reportado evidencias de validez aparente y de contenido, así como también, evidencias de validez basada en la estructura interna del instrumento. En efecto, se observó, mediante el AFE, un adecuado ajuste del modelo a los datos y a una estructura de tres factores (Atención, Claridad y Reparación Emocional), que describen el 54,5 \% de la varianza. Cada factor presenta al menos cuatro ítems y a su vez, todos los ítems presentan cargas factoriales superiores a .30. Estos resultados concuerdan con los reportados por Salovey et al. (1995) en la versión original de la escala, con los de la versión española (Fernández-Berrocal et al., 2004) y con los resultados del estudio psicométrico preliminar del TMMS-48 realizado por Mikulic et al. (2017).

En cuanto a las correlaciones entre los tres factores, los resultados mostraron que solo la correlación entre Claridad y Reparación Emocional fue moderada, mientras que Atención Emocional mostró una asociación débil tanto con Claridad como con Reparación Emocional. Estos resultados, no solo coinciden con los hallados en la versión española (Fernández-Berrocal et al., 2004), sino que se encuentran en la línea de lo esperado. De este modo, a diferencia de lo que sucede en el caso de Claridad y Reparación Emocional (donde altos puntajes serían indicadores de un adecuado ajuste emocional), en Atención Emocional, puntuaciones muy altas podrían eventualmente dar cuenta de un proceso de rumiación o hipervigilancia de las emociones y sensaciones, que conduciría a un desajuste emocional, perpetuando estados emocionales negativos (Extremera-Pacheco \& Fernández-Berrocal, 2005; FernándezBerrocal, Salovey, Vera, Ramos \& Extremera, 2017).

En relación con la consistencia interna, se verificaron coeficientes alfa de Cronbach y alfa ordinal, respectivamente, muy buenos para las tres sub-escalas (Atención: $\alpha=.82$ y $\alpha=.84$; Claridad: $\alpha=.84$ y $\alpha=.87$; y Reparación: $\alpha=$ .85 y $\alpha=.88$ ). Estos resultados se encuentran en concordancia con lo evidenciado en la escala original (Atención: $\alpha=.86$; Claridad: $\alpha=.88$; y Reparación: $\alpha=.82$ ) y en la versión española (Atención: $\alpha=.90$; Claridad: $\alpha=.90$; y Reparación: $\alpha=$.86) (Fernández-Berrocal et al., 2004; Salovey et al., 1995). 
Respecto a la relación con características sociodemográficas, no se observan diferencias estadísticamente significativas en ninguna de las sub-escalas en función del sexo y la edad, lo cual coincide no solo con la versión original y el estudio de validación de la versión española (Fernández-Berrocal et al., 2004; Salovey et al., 1995), sino también con estudios realizados en otros contextos culturales (Bonet \& Guillén, 2017; Cazalla-Luna \& Molero, 2016; Colorado, García, Alfonso, \& Ospino, 2012; del Carmen-Giménez-Espert \& PradoGascó, 2018; Gartzia, Aritzeta, Balluerka, \& Barberá, 2012). No obstante, varios trabajos han reportado diferencias significativas en función del sexo (Gómez-Núñez et al., 2018; López, Maciá, \& Juan, 2018; Rodríguez et al., 2019; Villacreces \& Achi, 2017), observándose, en general, mayores niveles de Atención Emocional en las mujeres y niveles más elevados de Regulación Emocional en los hombres (Merchán-Clavellino, Morales-Sánchez, MartínezGarcía, \& Gil-Olarte, 2018). Por lo tanto, dado que existen hasta la fecha resultados discordantes al respecto, continúa siendo un aspecto controvertido en el campo de estudio de la IE tanto en población general como en muestras de estudiantes. Es preciso que futuros estudios aborden y profundicen esta temática (Bonet \& Guillén, 2017; Rodríguez et al., 2019).

Con el fin de obtener evidencias de validez basada en la relación con otras variables, y debido a que varios estudios dan cuenta de la relación existente entre Inteligencia Emocional y Personalidad (van der Linden et al., 2017), se realizaron correlaciones entre las sub-escalas de IEA del TMMS-24 y las sub-escalas del cuestionario de personalidad AEP. Estas correlaciones han resultado estadísticamente significativas (excepto para Responsabilidad/ Reparación Emocional, Responsabilidad/Claridad Emocional, y Extraversión/ Claridad Emocional).

A diferencia de lo observado en un importante número de investigaciones previas, que evidencian que Atención correlaciona positivamente con todas las dimensiones de la personalidad, mientras que Claridad y Reparación correlacionan positivamente con Extraversión, Amabilidad, Responsabilidad y Apertura, y negativamente con Neuroticismo (Salguero, Fernández-Berrocal, Balluerka, \& Aritzeta, 2010; Leible \& Snell, 2004; Salovey et al., 1990), en el presente estudio, Atención y Reparación presentaron correlaciones negativas con Neuroticismo, mientras que Claridad presentó una correlación positiva con Neuroticismo.

A modo de hipótesis, cabría suponer que, en esta población de universitarios, niveles altos de Atención acompañados de una suficiente capacidad de Reparación podrían asociarse con bajos niveles de Neuroticismo. Asimismo, 
niveles altos de Claridad, en especial cuando no es acompañada de una suficiente capacidad de Atención y Reparación Emocional, podrían asociarse con malestar psicológico, emociones negativas e inestabilidad emocional.

De hecho, dado que el Neuroticismo implica la tendencia a experimentar emociones negativas (tales como enojo, tristeza, ansiedad) intensa y frecuentemente, altos niveles en esta variable podrían conducir a que los universitarios vean facilitada su capacidad para experimentar y entender claramente sus emociones (Claridad). No obstante, ello no necesariamente implica que puedan interrumpir y regular adecuadamente sus estados emocionales negativos y prolongar los positivos (Reparación).

Por otro lado, es preciso destacar algunas cuestiones en relación con la intensidad de las correlaciones entre IEA y personalidad, ya que conducen al debate acerca del solapamiento-independencia entre ambos conceptos, discutiéndose si las dimensiones de la IEA están más cercanas al concepto de personalidad que al de inteligencia (Davies, Stankov, \& Roberts, 1998).

Por un lado, existe una serie de investigaciones que muestran correlaciones moderadas y altas entre ambos constructos, siendo interpretadas como evidencia de solapamiento (Mestre-Navas \& Guil-Bozal, 2006; van der Linden et al., 2017). Estos estudios sostienen que las denominadas habilidades emocionales son en realidad rasgos de personalidad y ponen en discusión la utilidad aplicada de las medidas de IEA (van Rooy, Viswesvaran, \& Pluta, 2005). En esta línea de trabajo, se encuentran, por ejemplo, los estudios de Pérez-González y Sánchez-Ruiz (2014), al igual que los de van der Linden, Tsaousis y Petrides (2012), y Veselka, Schermer, Martin y Vernon (2010) quienes obtuvieron correlaciones alrededor de $r=.70$.

No obstante, existen otras investigaciones que, al igual que el presente estudio, reportan correlaciones bajas y moderadas entre las dimensiones de IEA y de personalidad, aportando evidencias en favor de la independencia de ambos constructos, destacando la existencia de relaciones entre ellos e, incluso, señalando que ciertas variables de personalidad podrían operar como predictoras del índice global de IEA (Arias-Gallegos, Infantes-Chávez, \& Ceballos-Canaza, 2016; James, Bore, \& Zito, 2012; Mayer et al. 2016). De hecho, se han establecido niveles máximos de correlación para dar cuenta de la independencia entre conceptos ( $r$ de entre .40 y .50). Correlaciones por encima de esos valores indicarían solapamiento entre los constructos teóricos (Mestre-Navas \& Guil-Bozal, 2006). Consecuentemente, al presentar correlaciones menores a $r=.43$, el presente estudio sería una importante 
contribución al debate antes mencionado, aportando evidencia en favor de la independencia de la IEA.

Desde un punto de vista teórico, el modelo de IE de Mayer, Caruso y Salovey hace especial énfasis en diferenciar conceptualmente la IE de la personalidad, considerando que los rasgos de personalidad implican disposiciones para el comportamiento, mientras que la inteligencia supone habilidades organísmicas para comportarse. Es decir, aunque un rasgo, como por ejemplo la extraversión, puede depender de las habilidades sociales o resultar en ello, es una preferencia de comportamiento más que una habilidad. Saber lo que siente otra persona, en cambio, es una habilidad mental. Tal conocimiento puede provenir del factor g, o ser algo independiente de él (Mayer \& Salovey, 1993). Por lo tanto, este modelo prescinde de conceptos próximos a la personalidad (Billings et al., 2014), lo cual resulta necesario para considerar a la IE como un nuevo tipo de inteligencia (Mayer, Salovey, Caruso, \& Sitarenios, 2001).

\section{LIMITACIONES Y LÍNEAS FUTURAS DE INVESTIGACIÓN}

Entre las limitaciones del presente estudio, se puede mencionar, en el plano metodológico, el haber utilizado una muestra incidental en la cual la población está representada únicamente por estudiantes universitarios, siendo mayor la cantidad de mujeres en comparación a los hombres. Por este motivo, la generalización de los resultados es limitada. Futuros estudios buscarán ampliar las características socioeducativas de la muestra.

Además, si bien la estructura factorial obtenida es concordante con la propuesta en la escala original y con la mayoría de las versiones adaptadas a otras culturas, sería conveniente realizar un análisis factorial confirmatorio para poder evaluar el ajuste del modelo y analizar su invarianza en distintos grupos que puedan resultar de interés, como por ejemplo, en función del sexo de los evaluados. No obstante, las propiedades psicométricas examinadas hasta el momento han mostrado ser satisfactorias, por lo cual el TMMS-24 resulta ser un instrumento válido y confiable para la medición de Inteligencia Emocional Autopercibida desde un enfoque intrapersonal en población universitaria marplatense. Los resultados obtenidos se suman a los evidenciados por diversos estudios que verifican el funcionamiento robusto del TMMS-24 en diferentes culturas, es decir, las propiedades psicométricas del instrumento se mantienen estables en los diferentes grupos culturales que se han examinado (Fernández-Berrocal et al., 2004). 
Desarrollar estudios psicométricos resulta de suma importancia dado la escasez de instrumentos debidamente adaptados a nuestro medio. Las adaptaciones locales permiten, entre otras cosas, realizar comparaciones válidas con los resultados obtenidos en otros países. Solo de esta manera se podrá determinar en qué medida las habilidades de las emociones llegan a moldearse por la influencia sociocultural.

Conflicto de interés: Los autores no presentan conflicto de intereses.

Financiamiento: Artículo derivado de un trabajo conjunto entre becarios del Consejo Nacional de Investigaciones Científicas y Técnicas (CONICET).

Agradecimientos: A todos los participantes de la presente investigación.

\section{REFERENCIAS}

Abad, F. J., Olea, J., Ponsoda, V., \& García, C. (2011). Medición en Ciencias Sociales y de la Salud. Madrid: Síntesis.

Aksöz, I.., Bugay, A., \& Erdur-Baker, Ö. (2010). Turkish adaptation of the trait meta-mood scale. Procedia-Social and Behavioral Sciences, 2(2), 2642-2646. https://doi.org/10.1016/j.sbspro.2010.03.387

American Educational Research Association [AERA], American Psychological Association [APA] y National Council on Measurement in Education [NCME] (2014). Standards for Educational and Psychological Testing. Washington, DC: AERA

American Psychological Association (2010). Ethical Principles for Psychologists and Code of Conduct. Washington, D.C.: APA.

Arias-Gallegos, W. L., Infantes-Chávez, A. E., \& Ceballos-Canaza, K. D. (2016). Personalidad, inteligencia emocional y estilos de afrontamiento en los conciliadores de la ciudad de Arequipa. Interacciones, 2(1), 21-32. http://revistainteracciones.com

Ato, M., López, J., \& Benavente, A. (2013). Un Sistema de clasificación de los diseños de investigación en psicología. Anales de Psicología 29(3), 1038-1059. http:// dx.doi.org/10.6018/analesps.29.3.178511

Barna, J., \& Brott, P. (2011). How important is personal-social development to academic achievement? The elementary school conselor's perspective. Professional School Counseling, 14(3), 242-250. https://doi.org/10.1177/2156759X1101400308

Bar-On R. (1997). BarOn Emotional Quotient Inventory: Technical Manual. Toronto: Multi-Health Systems.

Belmonte-Lillo, V. M. (2013). Inteligencia emocional y creatividad: Factores predictores del rendimiento académico (Tesis inédita de doctorado). Universidad de Murcia, Murcia, España.

Billings, C. E., Downey, L. A., Lomas, J. E., Lloyd, J., \& Stough, C. (2014). Emotional Intelligence and scholastic achievement in pre-adolescent children. Personality and Individual Differences, 65, 14-18. http://dx.doi.org/10.1016/j.paid.2014.01.017

Bonet, G. P., \& Guillén, L. A. V. (2017). Inteligencia emocional percibida (IEP) en el alumnado universitario de educación: análisis comparativo por género y grado. EA, Escuela Abierta: Revista de Investigación Educativa, (20), 23-34. http://dx.doi. org/10.29257/EA20.2017.03 
Bourdier, L., \& Ringuenet, D. (2016). Propriétés psychométriques de la version française d'une échelle de mesure de l'intelligence émotionnelle perçue: la Trait Meta-Mood Scale (TMMS). Canadian Journal of Psychiatry, 61(10), 1-11. https:// doi.org/10.1177/0706743716639936

Brackett, M. A., \& Mayer, J. D. (2003). Convergent, discriminant, and incremental validity of competing measures of emotional intelligence. Personality and Social Psychology Bulletin, 29(9), 1147-1158. https://doi. org/10.1177/0146167203254596

Brito-Costa, S., Castro, F. V., Moisao, A., Alberty, A., de Almeida, H., \& Fernández, M. I. R. (2016). Psychometric properties of Portuguese version of trait meta-mood scale (TMMS24). International Journal of Developmental and Educational Psychology INFAD Revista de Psicología, 2(1), 133-142. http://dx.doi.org/10.17060/ ijodaep.2016.n1.v2.296

Calero, A. (2013). Versión argentina del TMMS para adolescentes: una medida de la inteligencia emocional percibida. Cuadernos de Neuropsicología/Panamerican Journal of Neuropsychology, 7(1), 104-119. https://doi.org/10.7714/cnps/7.1.206

Cazalla-Luna, N., \& Molero, D. (2016). Inteligencia emocional percibida, disposición al optimismo-pesimismo, satisfacción vital y personalidad de docentes en su formación inicial. Revista de Investigación Educativa, 34(1), 241-258. http:// dx.doi.org/10.6018/rie.34.1.220701

Cejudo, J., López-Delgado, M. L., \& Rubio, M. J. (2016). Inteligencia emocional y resiliencia: su influencia en la satisfacción con la vida en estudiantes universitarios. Anuario de Psicología, 46(2), 51-57. Recuperado de https://www.redalyc. org/articulo.oa?id=97049408001

Colorado, Y., García, K., Alfonso, L., \& Ospino, G. (2012). Características de inteligencia emocional y género en estudiantes de psicología y administración de empresas de una universidad pública de Santa Marta, Colombia: un estudio piloto. Duazary, 9(2), 132-139. http://revistaduazary.unimagdalena.edu.co/

Davies, M., Stankov, L., \& Roberts, R. D. (1998). Emotional intelligence: in search of an elusive construct. Journal of Personality and Social Psychology, 75(4), 989-1015. https://psycnet.apa.org/search

del Carmen-Giménez-Espert, M., \& Prado-Gascó, V. J. (2018). Diferencias en los niveles de inteligencia emocional en función del sexo en enfermería. Revista de Investigación en Psicología Social, 2(1), 40-49. http://sportsem.uv.es/j_sports_ and_em/index.php/rips/article/viewFile/74/101

Delgado, B., Martínez-Monteagudo, M. C., Rodríguez, J. R., \& Escortell Sánchez, R. (2019). La autoeficacia académica y la inteligencia emocional como factores asociados al éxito académico de los estudiantes universitarios. Revista Gestión de las Personas y Tecnología, 12(35), 46-60. https://reunir.unir.net/ handle/123456789/9430

DeSteno, D., Gross, J. J., \& Kubzansky, L. (2013). Affective science and health: The importance of emotion and emotion regulation. Health Psychology, 32(5), 474-486. http://dx.doi.org/10.1037/a0030259

Domínguez-Lara, S. A., \& Medrano, L. A. (2016). Propiedades psicométricas del cuestionario de regulación cognitiva de la emociones (CERQ) en estudiantes universitarios de Lima. Psychologia. Avances de la Disciplina, 10(1), 53-67. http:// www.scielo.org.co/pdf/psych/v10n1/v10n1a06.pdf

Ekman, P., Sorenson, E. R., \& Friesen, W. V. (1969). Pan-cultural elements in facial displays of emotion. Science, 164, 86-88, http://dx.doi.org/10.1126/ science.164.3875.86 
Elfenbein, H. A., \& MacCann, C. (2017). A closer look at ability emotional intelligence (EI): What are its component parts, and how do they relate to each other?. Social and Personality Psychology Compass, 11(7), e12324. https://doi.org/10.1111/ spc3.12324

Extremera-Pacheco, N., \& Fernández-Berrocal, P. (2005). Inteligencia emocional percibida y diferencias individuales en el meta-conocimiento de los estados emocionales: una revisión de los estudios con el TMMS. Ansiedad y Estrés, 11(2-3), 101-122. http://www.elsevier.es/es-revista-ansiedad-estres-242

Extremera-Pacheco, N., \& Fernández-Berrocal, P. (2016). Inteligencia Emocional y Educación: Psicología. Valencia: Grupo 5.

Fernández, I., Carrera, P., \& Sánchez, F. (2001). Cultura, conocimiento social y comunicación de las emociones. En J.F. Morales, D. Páez, A. Kornblit y D. Asún (Eds.) Psicología Social (pp.265-285). Argentina: Prentice Hall.

Fernández-Berrocal, P., \& Extremera-Pacheco, N. (2008). A review of trait meta-mood research. International Journal of Psychology Research, 2(1/2), 39-67. https:// revistas.usb.edu.co/index.php/IJPR/index

Fernández-Berrocal, P., Alcaide, R., Domínguez, E., Fernández-McNally, C., Ramos, N. S., \& Ravira, M. (1998). Adaptación al castellano de la escala rasgo de metaconocimiento sobre estados emocionales de Salovey et al.: datos preliminares. En Libro de actas del V Congreso de Evaluación Psicológica (Vol. 1, pp. 83-84).

Fernández-Berrocal, P., Berrios-Martos, P., Extremera-Pacheco, N., \& Augusto, J. M. (2012). Inteligencia emocional: 22 años de avances empíricos. Behavioral Psychology, 20(1), 5-14. https://www.behavioralpsycho.com/wp-content/ uploads/2019/08/01.Fernandez-Berrocal_20-1.pdf

Fernández-Berrocal, P., Extremera-Pacheco, N., \& Ramos, N. (2004). Validity and reliability of the Spanish modified version of the Trait Meta-Mood Scale. Psychological Reports, 94(3), 751-755. https://journals.sagepub.com/home/prx

Fernández-Berrocal, P., Ruiz-Aranda, D., Salguero, J. M., Palomera, R. M., \& Extremera-Pacheco, N. (2018). The Relationship of Botín Foundation's Emotional Intelligence Test (TIEFBA) with Personal and Scholar Adjustment of Spanish Adolescents. Revista de Psicodidáctica, 23(1), 1-8. www.elsevier.es/psicod

Fernández-Berrocal, P., Salovey, P., Vera, A., Ramos, N., \& Extremera, N. (2001). Cultura, inteligencia emocional percibida y ajuste emocional: un estudio preliminar. Revista Electrónica de Motivación y Emoción, 4, 1-15. http://www.robertexto.com/archivo12/cultura_inteli_emo.htm

Gartzia, L., Aritzeta, A., Balluerka, N., \& Barberá, E. (2012). Inteligencia emocional y género: más allá de las diferencias sexuales. Anales de Psicología, 28(2), 567-575. http://doi.org/10.6018/analesps.28.2.124111

George, D., \& Mallery, M. (2016). IBM SPSS Statistics 23 Step by Step A Simple Guide and Reference (14th Edition). Boston, MA: Allyn and Bacon.

Giromini, L., Colombarolli, M. S., Brusadelli, E., \& Zennaro, A. (2017). An Italian contribution to the study of the validity and reliability of the trait meta-mood scale. Journal of Mental Health, 26(6), 523-529. https://doi.org/10.1080/09638 237.2017.1340621

Goleman, D. P (1995). Emotional intelligence: Why it can matter more than IQ for character, health and lifelong achievement. New York: Bantam Books.

Gómez-Núñez, M. I., Torregrosa, M. S., Inglés, C. J., Lagos San Martín, N. G., Sanmartín, R., Vicent, M., \& García-Fernández, J. M. (2018). Factor Invariance of the Trait Meta-Mood Scale-24 in a Sample of Chilean Adolescents. Journal of Personality Assessment, 1-7. https://doi.org/10.1080/00223891.2018.1505730 
Gorostiaga, A., Balluerka, N., Aritzeta, A., Haranburu, M., \& Alonso-Arbiol, I. (2011). Measuring perceived emotional intelligence in adolescent population: Validation of the Short Trait Meta-Mood Scale (TMMS-23). International Journal of Psychology and Psychological Therapy, 11(3), 523-537. http://www.redalyc.org/ articulo.oa?id=56019881007

Graesser, A. C. (2019). Emotions are the experiential glue of learning environments in the 21st century. Learning and Instruction, 101212. https://doi.org/10.1016/j. learninstruc.2019.05.009

Hair, J. F., Black, W. C., Babin, B. J., \& Anderson, R. E., (2009). Multivariate Data Analysis (7th edition). Upper Saddle River, NJ: Prentice Hall.

International Test Commission (2014, april 10). ITC Statement on the Use of Tests and Other Assessment Instruments for Research Purposes. http://www.intestcom. org/page/22

James, C., Bore, M., \& Zito, S. (2012). Emotional intelligence and personality as predictors of psychological well-being. Journal of Psychoeducational Assessment, 30(4), 425-438. https://doi.org/10.1177/0734282912449448

Kotsou, I., Mikolajczak, M., Heeren, A., Grégoire, J., \& Leys, C. (2019). Improving emotional intelligence: A systematic review of existing work and future challenges. Emotion Review, 11(2), 151-165. https://doi.org/10.1177/1754073917735902

Ledesma, R. D., Sánchez, R., \& Díaz-Lázaro, C. M. (2011). Adjective checklist to assess the big five personality factors in the Argentine population. Journal of Personality Assessment, 93(1), 46-55. https://doi.org/10.1080/00223891.2010.513708

Leible, T. L., \& Snell Jr, W. E. (2004). Borderline personality disorder and multiple aspects of emotional intelligence. Personality and Individual Differences, 37(2), 393-404. https://doi.org/10.1016/j.paid.2003.09.011

Li, C., Yan, J., Yin, X., \& Wu, Z. (2002). A primary study of the application of the Trait Meta-Mood Scale in military medical students. Chinese Journal of Clinical Psychology, 10, 202-203. https://www.safetylit.org/week/journalpage. php?jid=15389

López, R. S., Maciá, C. G., \& Juan, M. V. (2018). Inteligencia emocional en alumnado de formación profesional. Diferencias en función del curso, del género y de la edad. Educar, 54(1), 229-245. https://doi.org/10.5565/rev/educar.797

Lorenzo-Seva, U., \& Ferrando, P. J. (2015). Factor 10.3. España: Rovira I Virgili University.

Lloret-Segura, S., Ferreres-Traver, A., Hernández-Baeza, A., \& Tomás-Marco, I. (2014). El análisis factorial exploratorio de los ítems: una guía práctica, revisada y actualizada. Anales de Psicología, 30(3), 1151-1169. http://dx.doi.org/10.6018/ analesps.30.3.19936

MacCann, C., Jiang, Y., Brown, L. E., Double, K. S., Bucich, M., \& Minbashian, A. (2020). Emotional intelligence predicts academic performance: A meta-analysis. Psychological Bulletin, 146(2), 150. http://dx.doi.org/10.1037/bul0000219.supp

Mayer, J. D., \& Salovey, P. (1993). The intelligence of emotional intelligence. Intelligence, 17(4), 433-442. https://doi.org/10.1016/0160-2896(93)90010-3

Mayer, J. D., Caruso, D. R., \& Salovey, P. (1999). Emotional intelligence meets traditional standards for an intelligence. Intelligence, 27(4), 267-298. https://doi. org/10.1016/S0160-2896(99)00016-1

Mayer, J. D., Caruso, D. R., \& Salovey, P. (2016). The ability model of emotional intelligence: Principles and updates. Emotion Review, 8(4), 290-300. https://doi. org/10.1177/1754073916639667 
Mayer, J. D., Roberts, R. D., \& Barsade, S. G. (2008). Human abilities: Emotional intelligence. Annual Review of Psychology, 59, 507-536. https://doi.org/10.1146/ annurev.psych.59.103006.093646

Mayer, J. D., Salovey, P., Caruso, D. R., \& Sitarenios, G. (2001). Emotional intelligence as a standard intelligence. Emotion, 3(1), 232-242. https://doi.org/10.1037/15283542.1.3.232

Merchán-Clavellino, A., Morales-Sánchez, L., Martínez-García, C., \& Gil-Olarte, P. (2018). Valor predictivo de la inteligencia emocional rasgo en los estados afectivos: una comparación de género. Revista INFAD de Psicología. International Journal of Developmental and Educational Psychology, 1(1), 137-146. http:// infad.eu/RevistaINFAD/OJS/index.php/IJODAEP/article/viewFile/1178/1018

Mestre-Navas, J., \& Guil-Bozal, R. (2006). Medidas de ejecución versus medidas de autoinformes de inteligencia emocional. Ansiedad y Estrés, 12(2-3), 413-425. http://www.elsevier.es/es-revista-ansiedad-estres-242

Mikulic, I. M., Crespi, M., \& Caballero, R. (2018). Construcción de un inventario de inteligencia emocional percibida para adultos. Ciencias Psicológicas, 12(1), 121-136. http://dx.doi.org/10.22235/cp.v12i1.160

Mikulic, I. M., Crespi, M., Caballero, R. Y., Aruanno, Y., \& Paolo, A. M. (2017). Adaptación y validación del Trait Meta-Mood Scale (tmms-48) para su uso en adultos de Buenos Aires. IX Congreso Internacional de Investigación y Práctica Profesional en Psicología XXIV Jornadas de Investigación XIII Encuentro de Investigadores en Psicología del MERCOSUR. Facultad de Psicología - Universidad de Buenos Aires, Buenos Aires. https://www.aacademica.org/000-067/1102

Molero, P. P., Zurita-Ortega, F., Chacón-Cuberos, R., Castro-Sánchez, M., RamírezGranizo, I., \& Valero, G. G. (2020). La inteligencia emocional en el ámbito educativo: un meta-análisis. Anales de Psicología, 36(1), 84-91. https://doi. org/10.6018/analesps.345901

Otto, J. H., Döring-Seipel, E., Grebe, M., \& Lantermann, E. D. (2001). Development of a questionnaire for measuring perceived emotional intelligence: Attention to, clarity, and repair of emotions. Diagnostica, 47(4), 178-187. http://dx.doi. org/10.1026//0012-1924.47.4.178

Pérez-González, J. C., \& Sánchez-Ruiz, M. J. (2014). Trait emotional intelligence anchored within the Big Five, Big Two and Big One frameworks. Personality and Individual Differences, 65, 53-58. https://doi.org/10.1016/j.paid.2014.01.021

Peters, G. J. Y. (2014). The alpha and the omega of scale reliability and validity: Why and how to abandon Cronbach's alpha and the route towards more comprehensive assessment of scale quality. European Health Psychologist, 16(2), 56-69. http://www.ehps.net/ehp/index.php/contents

Petrides, K. V., \& Furnham, A. (2001). Trait emotional intelligence: Psychometric investigation with reference to established trait taxonomies. European Journal of Personality, 15, 425-448. http://dx.doi.org/10.1002/per.416

Petrides, K. V., Mikolajczak, M., Mavroveli, S., Sánchez-Ruiz, M. J., Furnham, A., \& Pérez-González, J. C. (2016). Developments in trait emotional intelligence research. Emotion Review, 8(4), 335-341. https://doi.org/10.1177/1754073916650493

Rincón, R. A., \& Rodríguez, Á. P. A. (2018). Validez y confiabilidad de la escala rasgo de metaconocimiento emocional (TMMS-24) en profesores universitarios. Revista Lebret, (10), 61-72. https://doi.org/10.15332/rl.v0i10.2197

Rodríguez, J., Sánchez, R. F., Ochoa, L. M., Cruz, I. A., \& Fonseca, R. T. (2019). Niveles de inteligencia emocional según género de estudiantes en la educación superior. Revista Espacios, 40(31). https://www.revistaespacios.com/ a19v40n31/19403126.html 
Salguero, J. M., Fernández-Berrocal, P., Balluerka, N., \& Aritzeta, A. (2010). Measuring perceived emotional intelligence in the adolescent population: Psychometric properties of the Trait Meta-Mood Scale. Social Behavior and Personality: An International Journal, 38(9), 1197-1209. https://doi.org/10.2224/sbp.2010.38.9.1197

Salovey, P., \& Mayer, J. D. (1990). Emotional intelligence. Imagination, Cognition and Personality, 9(3), 185-211. https://doi.org/10.1.1.385.4383\&rep=rep1\&type=pdf

Salovey, P., Mayer, J. D., Goldman, S. L., Turvey, C., \& Palfai, T. P. (1995). Emotional attention, clarity, and repair: Exploring emotional intelligence using the Trait Meta-Mood Scale. En J.W. Pennebaker (Ed.), Emotion, Disclosure, \& Health (pp. 125-154). Washington, DC, US: American Psychological Association. http://dx.doi. org/10.1037/10182-006

Sosa-Correa, M., Rodríguez-Ake, A., Ayuso, R., Ponce, N., \& Mestre, J. (2018). Propiedades psicométricas: escala Yucatán del uso percibido de la Inteligencia Emocional en menores (EYUPIE-M). Revista Iberoamericana de Diagnóstico y Evaluación-e Avaliação Psicológica, 3(48), 127-138. https://doi.org/10.21865/RIDEP48.3.11

Tabachnick, B. G., \& Fidell, L. S. (2001). Using Multivariate Statistics. New York: Harper \& Row.

Uriel, E., \& Aldas, J. (2005). Análisis Multivariante Aplicado. España: Thomson.

Valdivia-Vázquez, J. A., Rubio-Sosa, J. C. A., \& French, B. F. (2015). Examination of the Spanish Trait Meta-Mood Scale-24 Factor Structure in a Mexican Setting. Journal of Psychoeducational Assessment, 33(5), 473-482. https://doi. org/10.1177/0734282914552052

Van der Linden, D., Pekaar, K. A., Bakker, A. B., Schermer, J. A., Vernon, P. A., Dunkel, C. S., \& Petrides, K. V. (2017). Overlap between the general factor of personality and emotional intelligence: A meta-analysis. Psychological Bulletin, 143(1), 36-52. https://doi.org/10.1037/bul0000078

Van der Linden, D., Tsaousis, I., \& Petrides, K. V. (2012). Overlap between General Factors of Personality in the Big Five, Giant Three, and trait emotional intelligence. Personality and Individual Differences, 53(3), 175-179. https://doi.org/10.1016/j. paid.2012.03.001

Van Rooy, D. L., Viswesvaran, C., \& Pluta, P. (2005). An evaluation of construct validity: What is this thing called emotional intelligence?. Human Performance, 18(4), 445-462. https://www.tandfonline.com/loi/hhup20

Veselka, L., Schermer, J. A., Martin, R. A., \& Vernon, P. A. (2010). Relations between humor styles and the Dark Triad traits of personality. Personality and Individual Differences, 48(6), 772-774. https://doi.org/10.1016/j.paid.2010.01.01

Villacreces, J. A. T., \& Achi, V. H. Z. (2017). Aplicación del test TMMS-24 para el análisis y descripción de la Inteligencia Emocional considerando la influencia del sexo. Revista Publicando, 4(11), 162-181. file:///C:/Users/rochi/OneDrive/Escritorio/518-2039-1-PB.pdf

Zafra, E. L., Martos, M. P., \& Martos, P. B. (2014). Adaptación y validación al Español del EQ-i en universitarios. Boletín de Psicología, 110, 21-36. https:// www.uv.es/seoane/boletin/boletin.html

Esta obra está bajo: Creative commons attribution 4.0 international license. El beneficiario de la licencia tiene el derecho de copiar, distribuir, exhibir y representar la obra y hacer obras derivadas siempre y cuando reconozca y cite la obra de la forma especificada por el autor o el licenciante.

\section{(cc) BY}

